\title{
Study of Manufacturing Logistics Outsourcing Decision
}

\author{
Zhengfa Yang and Hua Li \\ Institute of Economic and Trade, Jiangxi University of Technology, Nanchang 330098, China
}

Keywords: Enterprise Logistics; Outsourcing Decision; AHP

\begin{abstract}
No matter at home or abroad, logistics business outsourcing has reached certain scale. With the promotion and application of supply chain management theory in enterprise, the trend of logistics business outsourcing will be further strengthened. The object of logistics outsourcing gradually changes from single function to multifunction, from simple to complex development; third-party logistics business gradually expands outwards from link of material supply between upstream and downstream enterprise, from storage and transport gradually developed into order management, customer relationship management, information system management, inventory management, management consulting and other aspects. To make use of the logistics outsourcing to improve the production of enterprise and value of service activities, to enhance competitiveness of enterprise, the enterprises shall give priority to a right outsourcing decision.Manufacturing enterprise' choosing logistics outsourcing is becoming a trend, and how to ensure the smooth progress of logistics outsourcing has also become a problem that manufacturing enterprise must consider. At present, manufacturing enterprises are less satisfied with logistics outsourcing service providers, logistics outsourcing service providers need to enhance the service level to meet the needs of outsourcing party. At the same time, manufacturing enterprises also need to strengthen the management of service provider to promote their logistics activities in accordance with requirements of enterprise. An appropriate third-party logistics service provider becomes the most important step for manufacturing enterprise to successfully manage service provider, especially under the actual condition that the level of third-party logistics service provider of China is good and bad mixed together, and it seems particularly important for manufacturing enterprise to choose. If manufacturing logistics outsourcing is used properly, it can reduce the logistics cost of enterprise, share risks, and enhance core competitiveness; otherwise, the companies will face many risks.
\end{abstract}

\section{Overview}

Logistics outsourcing refers to that in order to concentrate resources and energy on their core business and enhance core competitiveness of enterprise, production enterprise, sales enterprise or other enterprise, entrust some or all of the logistics businesses which they are not good at or have no advantage in to professional third-party logistics enterprise for operation by way of contract. In the market technology environment of rapid development, through outsourcing logistics business, manufacturing enterprise can establish a strategic alliance with third-party logistics service cooperation enterprise, use its superior resources, shorten the time of product development, production and sales, lighten the product risks caused by change in technology and market demand; meanwhile, it also improves the reflection speed of supply chain, so manufacturing enterprise can respond quickly to changes of market demand, and increase the supply chain flexibility. 


\section{Study of manufacturing enterprise logistics outsourcing decision risk}

Source of manufacturing enterprise's logistics outsourcing decision risk. Logistics outsourcing is a business combination, which is made up of enterprises with different core capabilities and logistics service providers and driven by interests. It is an open and complex system. The interaction between commission enterprise and agency enterprise has greater complexity, coupled with human error, system constraint, backward ideas, technical management defects and other factors. In the process of mutual activities, many potential sources of risk will appear in environment, personnel, organization, decision-making, cultural and other aspects.

Internal environmental factors and external environmental factors of manufacturing enterprise. Logistics outsourcing risk generated by internal environment factors of manufacturing enterprise is mainly reflected in: the corporate culture is different and boycott from internal staff. External environment triggering enterprise logistics outsourcing risk mainly refers to the adverse effects on enterprise logistics outsourcing caused by natural environment, political and economic environment, market environment, legal environment and other factors,

Outsourcing process management factors. In the process of enterprise outsourcing decision and implementation, because of the management problems of the enterprise itself, it will lead to outsourcing risk, there are about the following points: (1) outsourcing decision mistakes; (2) logistics service provider is too single; (3) unstable cooperation; (4) not reaching consensus, a lot of practice demonstrate that, not reaching consensus by both parties of cooperation is the most common cause of third-party logistics cooperation failure; (5) outsourcing contract is not standardized; (6) lack of reasonable performance evaluation.

Information transfer factor. (1) Imperfection of information communication and feedback mechanism. (2) Information asymmetry and incomplete information.

\section{Problems needing attention in logistics outsourcing decision of manufacturing enterprise}

Selection of outsourcing partners. A very important question in outsourcing decision is to whom researchers outsource, i.e. selection of outsourcing partner. First, for external potential logistics provider, researchers should survey the cost of the project, advantage line, evaluate its long-term viability, evaluate its credibility, etc. Especially for commitment and quotation by logistics provider, researchers must carefully analyze the measure. Offer is not only a total sum, and it should include cost details of every job. Logistics outsourcing commitment must come from top management of logistics provider, and avoid inconsistencies in understanding relevant clause in fulfilling contract. On the basis of evaluation, compare potential multiple logistics outsourcing partners, and choose the outsourcing partners best meeting the needs of companies.

Control of logistics outsourcing activities. After manufacturing enterprise selects third-party logistics service provider, the enterprise should sign a corresponding carriage contract to define rights and obligations of both parties, minimize the risk of the company. Monitoring and controlling on outsourcing activity is important guarantee for smooth implementation of outsourcing. Even company signs agreement with logistics provider, performance of logistics provider should also be monitored, while giving them business information they need. Enterprise and third-party should pay attention to the mutual communication between them, compiling operation specification.

Taking "Win-win" as principle, consolidate partnership. Enterprise logistics outsourcing means that the benefit of enterprise and logistics service provider is bundled together, good 
partnership will benefit both sides, and bad act of either side will make both sides suffer. When choosing a third-party logistics provider, company should treat logistics outsourcing with long-term strategic thinking, by outsourcing, it does not only maximize company interests, but help logistics provider develop sustainably and stably, to achieve win-win situation. Therefore, mutual trust and loyalty between supply and requisitioning parties, fulfillment of commitment are key factors for the establishment of good outsourcing cooperation relation. Only in this way, the two sides can cooperate for a long term.

\section{Establishment of Logistics Service Provider Evaluation Index System of Manufacturing Enterprise}

Setting principles of logistics service provider evaluation index system of manufacturing enterprise. The evaluation index system of manufacturing enterprise logistics service provider should be designed to meet basic principles, such as comprehensive content, scientific and practical, objective and fair, strong operability and adaptability, including the following seven aspects:

(1) Principle of purposefulness. The purpose of designing supplier evaluation index system is to measure the competitiveness of supplier, to elect provider suitable for enterprise supply chain management, to establish strategic cooperative partnership, enhance competitiveness of enterprise supply chain.

(2) Principle of scientificity. Supplier evaluation index should accurately reflect the actual situation, which is conducive for enterprise to have fair, objective and comprehensive evaluation of supplier through evaluation.

(3) Principle of comprehensiveness. Evaluation index system must fully reflect the current comprehensive level of provider enterprise, and include various indexes of business prospects. In addition, the system should consider external economic environment, the index system should include not only the "hard" index which reflects strength of supplier, but also include "soft" index which reflects other competitive advantages of supplier.

(4) Principle of effectiveness. Effectiveness principle is the principle of performance, design of evaluation index system should take into account to create maximum output with minimum investment, economic benefit should be in an important position in evaluation index system, which requires the design of index system to try to simplify and stress the key point, so that the index system is easy to operate and feasible in practice.

(5) Principle of flexible operability. Evaluation index should be flexible enough to enable enterprise flexibly use index according to their characteristics and actual situation.

(6) Principle of combination of quantitativeness and qualitativeness. Competitiveness level of supply chain is an abstract concept, when doing comprehensive evaluation of competitiveness of supplier, quantitativeness index and qualitativeness index impacting competitiveness of supplier should be considered comprehensively, clarify the meaning of qualitativeness index and in accordance with certain criteria assignment, make qualitativeness index appropriately reflect the nature of index, quantitativeness index and qualitativeness index must have clear concept and precise calculation.

Establishme nt of enterprise logistics service provider evaluation index system. For enterprise, the aim of choosing to develop partnership with supplier is to improve the competitiveness of their supply chain, so when choose to evaluate their partner type supplier, index of all aspects affecting cooperation and competitiveness should be taken as key consideration. Accord ing to different levels, 
supplier evaluation can be divided into three aspects: supplier's credibility and popularity, evaluation of supplier technical capability, evaluation of supplier delivery capacity. Of course, there are many other indexes which can be used as evaluation basis of supplier, such as financial capacity, geographical location, corporate culture, and so on.

This article combines existing research results, based on specific investigation in enterprise and extensive consultation of advice of business management personnel, according to the principle of establishing evaluation index system, through repeated deliberation, finalizing three main factors influencing selection of supplier, including: corporate reputation and popularity, technology and delivery capability. Each impacting factor is set to be the first level index, and a number of evaluation indexes included under the first level index are set to be the secondary level index, constituting a complete supplier evaluation index system.

\section{Establishment of logistics Outsourcing Decision Model}

Definition and Application Conditions of AHP. Definition of AHP. The so-called AHP (AHP is short for Analytic Hierarchy Process, it refers to taking a complex multi-objective decision-making problem as a system, the target is decomposed into multiple targets or criteria, and then decomposed into a number of levels of multiple indexes (or criteria, constraints), level single sorting (weight) and total sorting is calculated by qualitative index fuzzy quantification method, by which it is taken as system method of target (multi-index), multi-program optimization decision-making. Its characteristic is that by dividing various elements in complex problems to mutually relative orderly level to make them in proper arrangement, according to subjective judgment structure (mainly pairwise comparison) on certain objective reality, combine expert advice and analysts' objective judgment results directly and effectively, describe quantitatively the importance of pairwise comparison of each level. Then, using mathematical method for calculating the weight reflecting relative importance sequence of elements in each level, by the total sorting of all the levels, calculate relative weight of all the elements and sorted.

Application conditions of AHP. AHP is applicable for multi-objective decision-making, it is used for the case where there are multiple impacting indexes, evaluate advantage and disadvantage of each program. When a decision is influenced by multiple factors, and there is hierarchical relationship between various elements or have obvious category, while degree of impact of each index on final evaluation can not be directly quantified with sufficient data, you can use AHP.

The application premise of AHP. When applying AHP, the following premises must be met:

(1) Elements of each level must be known, method and structure should be distinct, and they can be arranged as per hierarchical distinction.

(2) In the same level, relationship between various elements is equal, elements are independent, and there is no significant correlation.

(3) Index at the bottom can be quantified and can be measured by certain method.

(4) You need to be clear with affecting relationship of elements between the levels.

Establishment of logistics outsourcing decision model based on AHP

(1) To make clear problem.

(2) To establish hierarchy structure model.

(3) Structure judgment matrix.

(4) Level single-sorting and consistency checkout.

(5) The total sorting of level

(6) Consistency test of the total sorting of level 


\section{Conclusions}

Logistics outsourcing is a kind of development trend, and it is important means and method rapidly developed in the process of enterprise growth.

But researchers cannot blindly believe that outsourcing is a panacea to cure all diseases. With the logistics outsourcing increasingly getting attention from academic and enterprise today, researchers have to start from the actual condition of enterprise, to choose outsourcing model suitable for us, choose appropriate supplier.

By using AHP, this article focuses on the analysis of manufacturing company on how to choose the right supplier in logistics outsourcing and logistics outsourcing risk prevention and avoidance and other decision-making issues. In the model, this paper uses the method of combining qualitative research and quantitative research, and overcomes certain disadvantages, which is for the effective conduct of enterprise logistics outsourcing decisions, reduce cost and time of decision-making, and it has certain applicability and operability in enterprise logistics outsourcing practice.

\section{Acknowledgements}

This work was financially supported by the research result of the key construction disciplines, Industry Economics in Jiangxi University of Technology

\section{References}

[1] Zhou Yun. Current situation and trend analysis of third party logistics in China[J], Value Engineering, 2013, (327): 35-36.

[2] Zhu Youming. Situation and countermeasure analysis of logistics outsourcing of manufacturing enterprise of China [J], Chinese market, 2013 (11): 102-104.

[3] Geng Hongshan. From supply chain to see manufacturing enterprise logistics outsourcing [J], E-commerce, 2013 (1): 51-53.

[4] Bai Xiaojuan. Logistics outsourcing mechanism and model study of manufacturing enterprise[D], Beijing: Beijing Jiaotong University, 2014,19-24.

[5] Wu Qi. Logistics outsourcing motivation and profitability initiatives of Chinese manufacturing enterprise[J], Business research, 2014, (11): 3-4.

[6] Zhao Yingxia. Construction of logistics outsourcing provider evaluation index system of manufacturing enterprise[J], Foreign trade practice, 2013 (6): 24-26.

[7] Shen Liuyin. Driving force analysis and service provider selection research of manufacturing enterprise logistics service outsourcing[D], Shandong: Shandong Li Dong University, 2014,14-20.

[8] Zhang Xiaosheng. Risk of manufacturing enterprise logistics outsourcing and preventive measures[J], Business research, 2014, (8): 3-4.

[9]Gu Wenqi. Risks and countermeasures of enterprise logistics outsourcing[J], Management observation, 2013, (1): 35-36. 